\title{
Comparison between quantitative and qualitative biochemical markers in the diagnosis of acute coronary syndrome
}

\author{
Samir B Al-Mukhtar*, Akram J Ahmad** \\ Departments of biochemistry, * Ninevah College of Medicine, ${ }^{* *}$ Mosul College of Medicine, University of \\ Mosul \\ \begin{tabular}{ll} 
Received $\quad$ Accepted \\
\hline $6.2 .2011 \quad 12.6 .2011$
\end{tabular}
}

ABSTRACT

Objectives: To compare between the qualitative estimation of biochemical markers Point-of-Care testing) with the quantitative estimation of the same markers in the diagnosis of acute coronary syndrome (ACS).

Design: Case-series study.

Setting: This study was carried out in coronary care unit in Ibn- Sena Teaching Hospital in Mosul city from January to November, 2008.

Participant: Sixty five patients with acute coronary syndrome (ACS) presented with chest pain.

Main outcome measures: Three cardiac markers (Creatine kinase (CK-MB) activities (marker of necrosis), myoglobin (marker of muscle injury), and troponin I (marker of necrosis), were estimated qualitatively (near the patient $=$ Point-of-Care testing), and quantitatively, and the results were compared. Kappa test was used for the association between the quantitative and qualitative test results.

Results: The case-series study showed correlation of attributes between qualitative estimation results of troponin I, myoglobin and CK-MB and quantitative estimation results of the same parameters in $(88.9 \%)$ tests.

Conclusion: The early diagnosis of ACS might be facilitated by the use of qualitative point-of-care testing based on CK-MB, troponin I and myoglobin tests.

Keywords: Troponin I, myoglobin, CK-MB activity, Point-of-Care Testing, acute coronary syndrome, biochemical markers.

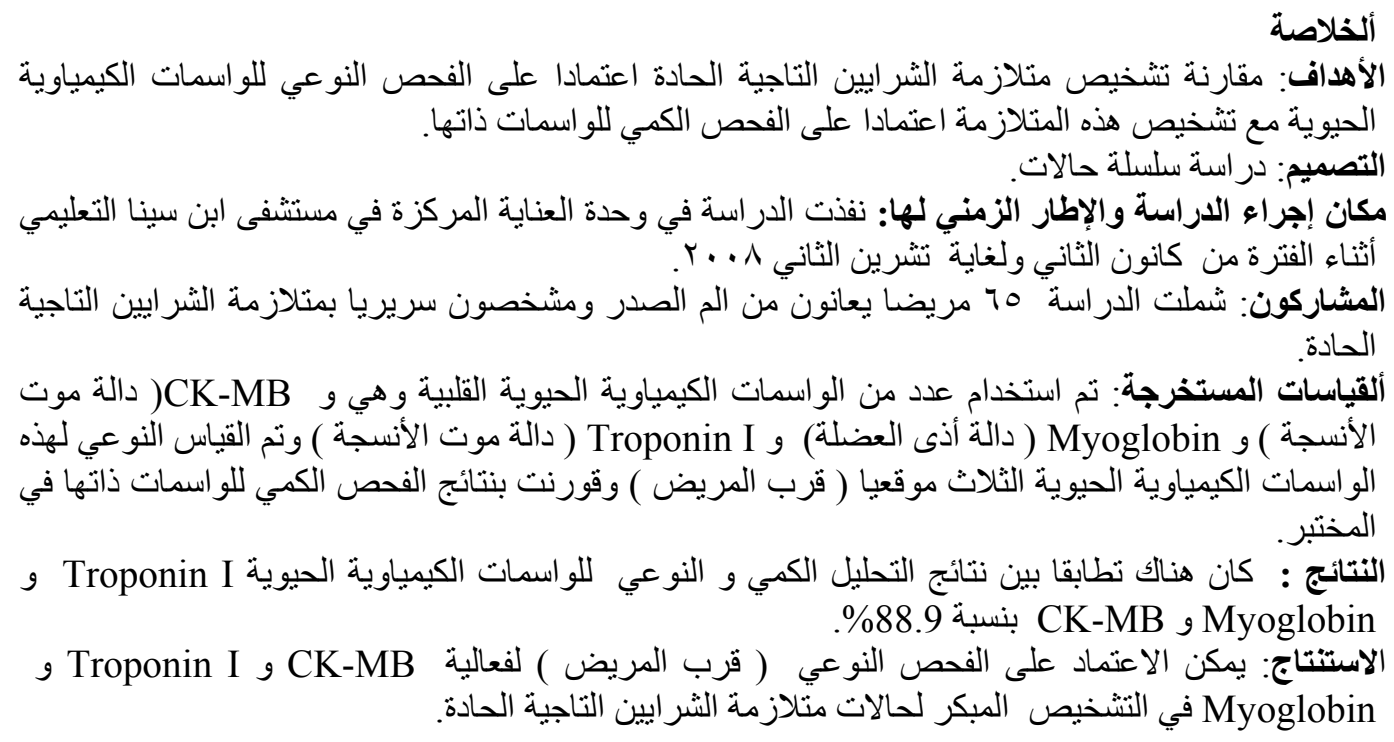


$\mathbf{S}_{\text {ymptoms and signs suggestive of }}$ acute myocardial infarction (AMI) and unstable angina which constitute acute coronary syndrome (ACS) are non specific and have low sensitivity for diagnosis of this condition ${ }^{1}$. The World Health Organization (WHO) definition and diagnosis of AMI, is currently two out of three: characteristic chest pain, diagnostic electrocardiogram changes and elevation of the biochemical markers in the blood samples ${ }^{2}$.

The electrocardiogram (ECG) may never show the classical features of ST elevation and new Q waves. Hence, in the early stage, there is no enough evidence in these patients for clear diagnosis and risk stratification ${ }^{3}$.

Biochemical marker identification which is sensitive and specific for myocardial ischemia and can easily and rapidly measured in serum would be clinically valuable ${ }^{4}$. Elevated levels of cardiac markers: Creatine kinase (CK-MB) activities (marker of necrosis), in addition to troponin I (marker of necrosis), and myoglobin (marker of muscle injury), could be useful in early diagnosis of acute coronary syndrome when patients admitted to coronary care unit $(\mathrm{CCU})^{5}$.

Point-of-care (POC) testing or "near patient" testing is defined as "any test that is performed at the time at which the test result enables a decision to be made and an action taken that leads to an improved health outcome" ${ }^{6}$. Point-of-care have been developed to detect CK-MB mass, myoglobin, cardiac troponin $\mathrm{I}$ and troponin $\mathrm{T}$ in small specimens of whole blood ${ }^{7}$. The POC is accurate, precise, rapid (fast turnaround time), easy to sample, easy to operate, with low calibration demands, disposable (used once) or easy to maintain, inexpensive and improves clinical decision-making ${ }^{8}$.

The aim of the study was to investigate the difference in the number of ACS diagnoses based on qualitative Point-of-Care (POC) testing and quantitative testing of patients compared with discordant diagnoses.

\section{Subjects and Methods}

This study was carried out in Ibn-Sina Teaching Hospital in Mosul during the period from January to November, 2008. It was carried out on 65 patients with chest pain in whom the diagnosis of ACS was within 12 hours of symptoms inception.

All patients who admitted to the $\mathrm{CCU}$ were with provisional diagnosis of ACS. The patients were looked for risk factors-"Smokers" were defined as patients currently smoking at the time of admission; "hypertension " was defined by self-report of a diagnosis and use of an anti-hypertensive medication, or if systolic blood pressure $>140 \mathrm{mmHg}$ or if diastolic blood pressure $>90 \mathrm{mmHg}^{9}$; "diabetes" as patients on insulin or taking oral hypoglycemic agents ${ }^{10} . "$ hypercholesterolemia "as total cholesterol of $>5.0 \mathrm{mmol} / \mathrm{L}$ on admission $^{11}$. A family history for ischemic heart disease (IHD) is considered positive if relatives have experienced an AMI prior to the age of 50 in men, and 55 in women ${ }^{1}$. History of ischemic heart diseases is defined as any group of acute or chronic cardiac disabilities resulting from insufficient supply of oxygenated blood to the heart, or is a group of diseases characterized by reduced blood supply to the heart muscle usually due to coronary artery disease (atherosclerosis of coronary arteries) ${ }^{12}$. Male gender and obesity were also considered as risk factors ${ }^{1}$.

All patients had cardiac markers tested within 12 hours post chest pain. Their ECG was assessed and any ischemic or progressive changes were documented $^{13}$. Body mass index was 
also taken. The study was carried out with the cooperation of senior cardiologist in CCU.

On admission, patients satisfying two out of the three criteria of WHO were considered as having myocardial infarction $^{2}$. The definitive diagnosis of myocardial infarction required all three criteria to be satisfied. Patients with chest pain and non-Q ECG pathology but not cardiac enzyme changes were diagnosed as having unstable angina ${ }^{14}$.

The study received the agreement of the Ethical committee of Ninevah Governorate Health Department and approval of Mosul College of medicine- Postgraduate Studies Committee. Subjects were informed about the purpose of the study and oral consent was obtained from the patients after the study had been explained to them.

A blood sample was immediately obtained for a qualitative bedside test with a ' 3 in 1 ' combination test (myoglobin, CKMB and troponin I) using the One-Step Immunoassay (NANOGEN Point of care, Toronto, Ontario. Canada). The POC testing of myoglobin, troponin I and CK-MB activity were done within 15 minutes and then compared with the quantitative estimation of the same parameters where the blood sample was obtained (at the same time) and were done later in the laboratory as discussed below. Utilizing the recommended cut-off values for the individual assays, the results of these 2 sets of tests were evaluated based on whether they were positive or negative for ACS. The diagnosis of AMI was based if two of the three parameters were positive ${ }^{15}$.

Venous blood samples $10 \mathrm{ml}$ were collected from patients on admission to the CCU. The blood samples were collected in vials. Blood allowed to clot fully by leaving for 15 minutes in water bath at $37{ }^{\circ} \mathrm{C}$, then serum was separated by centrifugation at 3000 rpm for 10 minutes. The serum was divided into two aliquots and stored at $-20{ }^{\circ} \mathrm{C}$. The first aliquot was for analysis of CK-MB activity by using BIOLABO CK- MB activity UV method (in the department of biochemistry) ${ }^{(16)}$. The second aliquot was for analysis of troponin I and myoglobin using ELISA monoclonal antibody (Biochek, Foster City, USA) for both parameter ${ }^{17,18}$.

The upper reference limits for the quantitative cardiac markers used in this study were: CK-MB activity $\geq 25$ IU ; Troponin $\geq 0.4 \mathrm{ng} / \mathrm{mL}$ and myoglobin $\geq 54.5 \mathrm{ng} / \mathrm{mL}$.

Kappa test was used for the association between the qualitative and quantitative test results. Kappa test is the reliability statistical test that measure the agreement beyond chance. The Kappa agreement, 1-20\% slight agreement, 21-40\% fair agreement, 41$60 \%$ good agreement, $61-80 \%$ substantial agreement, $81-99 \%$ almost perfect agreement ${ }^{(19)}$. All results were considered significant at $p$ equal to 0.05 or less.

\section{Results}

A total of 65 patients with ACS were examined and comparison between the rapid test (POC Testing) and the established laboratory based method showed sufficient agreement of results.

Description of study sample

As shown in Table 1, males constituted $58.5 \%$ of the study sample and $58.4 \%$ of cases were less than 60 years old. Moreover $69.2 \%$ of cases were overweight and obese. 
Table 1. Demographic characteristics of the studied groups

\begin{tabular}{|c|c|c|c|}
\hline Characteristics & & No. & $\%$ \\
\hline \multirow[t]{2}{*}{ Gender } & Male & 38 & 58.5 \\
\hline & Female & 27 & 41.5 \\
\hline \multicolumn{2}{|l|}{ Male/Female ratio } & \multicolumn{2}{|c|}{1.41} \\
\hline \multirow{5}{*}{ Age (years) } & $<50$ & 19 & 29.2 \\
\hline & $50-59$ & 19 & 29.2 \\
\hline & $60-69$ & 24 & 37.0 \\
\hline & $>70$ & 3 & 4.6 \\
\hline & Mean \pm SD & \multicolumn{2}{|c|}{$55.56 \pm 11.3$} \\
\hline \multirow{4}{*}{ BMI (kg/m2) } & Normal (18.5-25) & 20 & 30.8 \\
\hline & Overweight (25-30) & 28 & 43.1 \\
\hline & Obese $(>30)$ & 17 & 26.1 \\
\hline & Mean \pm SD & \multicolumn{2}{|c|}{$27.38 \pm 3.3$} \\
\hline
\end{tabular}

Frequency distribution of the risk factors among the studied patients Hypertension was associated with $54.4 \%, 52.3 \%$ had previous attack of
IHD and were smokers, 50.8\% were diabetic and had hypercholesterolemia and $29.2 \%$ reported positive family history of IHD (Table 2). 
Table 2. Frequency distribution of the studied patients according to risk factors

\begin{tabular}{|c|c|c|c|}
\hline Risk factors & & No. & $\%$ \\
\hline Family history of IHD & Yes & 19 & 29.2 \\
\cline { 2 - 4 } & No & 46 & 70.8 \\
\hline Previous history of IHD & Yes & 34 & 52.3 \\
\cline { 2 - 4 } & No & 31 & 47.7 \\
\hline \multirow{2}{*}{ Smoking } & Yes & 34 & 52.3 \\
\cline { 2 - 4 } & No & 33 & 50.8 \\
\hline Diabetes mellitus & Yes & 32 & 49.2 \\
\cline { 2 - 4 } & No & 36 & 54.4 \\
\hline Hypertension & Yes & 32 & 49.2 \\
\hline Hypercholesterolemia & No & 33 & 50.8 \\
\cline { 2 - 4 } & Yes & No & \\
\cline { 2 - 4 } & & & 32 \\
\hline
\end{tabular}

\section{Comparison}

As shown in table 3, the number of patients with positive results depending on quantitative estimation of troponin I was 42 while number of patients with positive results depending on qualitative method was 40. On the other hand number of patients with negative results depending on quantitative estimation of troponin I was 23 while number of patients with negative results depending on qualitative method was 25. The same commend is applicable for myoglobin and CK-MB activity in table 4 and 5 respectively.

Using Kappa test for comparison, troponin I was of the highest value among others (Kappa $=0.865$ ), followed by myoglobin and CK-MB (Kappa $=0.792$ and Kappa $=0.693$ respectively). This means that troponin $\mathrm{I}$ is the best test among the 3 tests (Tables 3, 4 and 5).

A correlation of attributes between qualitative (troponin I, myoglobin and CK-MB activity) and quantitative estimation of the same parameters was observed in $88.9 \%$ tests. 
Table 3. Kappa test for association between quantitative and qualitative serum troponin I

\begin{tabular}{|c|c|c|c|c|c|c|c|c|}
\hline \multirow[t]{2}{*}{ Method } & \multicolumn{2}{|c|}{ +ve } & \multicolumn{2}{|c|}{-ve } & \multicolumn{2}{|c|}{ Total } & Kappa & value $p$ - \\
\hline & No. & $\%$ & No & $\%$ & No & $\%$ & & \\
\hline Quantitative & 42 & 64.6 & 23 & 35.4 & 65 & 100 & & \\
\hline Qualitative & 40 & 61.5 & 25 & 33.5 & 65 & 100 & & \\
\hline
\end{tabular}

Table 4. Kappa test for association between quantitative and qualitative serum myoglobin

\begin{tabular}{|c|c|c|c|c|c|c|c|c|}
\hline \multirow{2}{*}{ Method } & \multicolumn{2}{|c|}{$+\mathrm{ve}$} & \multicolumn{2}{c|}{-ve } & \multicolumn{2}{c|}{ Total } & Kappa & value $p$ - \\
\cline { 2 - 9 } & No. & $\%$ & No & $\%$ & No & $\%$ & & \\
\hline Quantitative & 63 & 96.9 & 2 & 3.1 & 65 & 100 & \multirow{2}{*}{0.792} & $<0.001$ \\
\hline Qualitative & 61 & 93.8 & 4 & 6.2 & 65 & 100 & & \\
\hline
\end{tabular}

Table 5. Kappa test for association between quantitative and qualitative serum CKMB activity

\begin{tabular}{|c|c|c|c|c|c|c|c|c|}
\hline \multirow{2}{*}{ Method } & \multicolumn{2}{|c|}{+ ve } & \multicolumn{2}{|c|}{-ve } & \multicolumn{2}{c|}{ Total } & Kappa & value $p$ - \\
\cline { 2 - 9 } & No. & $\%$ & No & $\%$ & No & $\%$ & & \\
\hline Quantitative & 8 & 12.3 & 57 & 87.7 & 65 & 100 & \multirow{2}{*}{0.693} & $<0.001$ \\
\hline Qualitative & 7 & 10.7 & 58 & 89.3 & 65 & 100 & & \\
\hline
\end{tabular}




\section{Discussion}

Without a doubt, POC testing has become a critical component of laboratory medicine. More than 31,000 laboratory testing instruments and devices have been categorized by the FDA (Food and Drugs Association) under CLIA (Clinical Laboratory Improvement Amendment), and POC testing is now thought to represent $25 \%$ of the total expenditures of laboratory testing dollars. Its role in the management of patients is beneficial ${ }^{20}$.

As far as we are aware this is the first study in our locality, including Iraq and neighboring countries to compare the qualitative and quantitative measurement of cardiac biochemical markers.

In this case-series study a rapid qualitative POC testing of myoglobin, troponin and CK-MB activity were performed and then compared with the quantitative estimation of the same parameters as Central Laboratory Testing (CLT). The diagnosis of AMI was based if two of the three parameters were positive ${ }^{20}$. The use of multiple point-of-care cardiac marker assays has been reported in studies of patients with probable $\mathrm{ACSs}^{21,22}$.

In the present study, cardiac troponin I showed the best result among other markers $($ Kappa $=0.865)$ after which myoglobin was better than CK-MB in qualitative POC testing (Kappa $=0.792$ and 0.693 respectively) (Table 3, 4 and 5, respectively). This is consistent with other studies ${ }^{23-25}$. A correlation of attributes between qualitative (troponin I, myoglobin and CK-MB) and quantitative estimation of the same parameters was observed in $88.9 \%$ tests. This is in agreement with other studies ${ }^{25-28}$.

The qualitative assay allows (for example) the detection of troponin I in concentrations above the cut-off level. Meticulous observance of manufacturer's rules is imperative. A single preclinical rapid assay does not allow excluding AMI. However, the test enables one to identify patients who are at risk of dying from acute coronary syndrome ${ }^{(29)}$.

\section{Conclusion}

Point-of-care testing (qualitative) utilizing a panel of 3 cardiac markers has comparable diagnostic precision to the presently utilized testing strategy for AMI, with earlier availability of results even in the hand of physicians working in CCU outside the laboratory. Its correlation of attributes to quantitative assay is $88.9 \%$. Moreover, the results of elevated biochemical markers levels were obtainable within approximately 15 minutes of obtaining the blood specimen when using this POC system, allowing us to translate these findings into earlier treatment or triage.

\section{References}

1. Bloomfield P, Bradbury A, Grubb NR, et al. Cardiovascular diseases. In: Davidson's Principles and Practice of Medicine. 20th ed.. Edinburgh: Churchill Livingstone. 2006; P 519-646.

2. World Health Organization. Report of the Joint of Cardiology /World Health Organization task Force on Standardization of Clinical Nomenclature. Nomenclature and criteria for diagnosis of ischemic heart disease. Circulation 1979 ; 59: 607-9.

3. Panteghini M. Role and importance of Biochemical markers in clinical cardiology. Eur Heart J 2004; 25(14): 1187-96.

4. Panteghini M. Biochemical markers in acute coronary syndrome. Lab. Medica International 2003; 20(6): 6-7,112.

5. Kemp M, Donovan J, Higham H, et al. Biochemical markers of 
myocardial injury. B J A 2004; 93(1):63-73.

6. Price CP, John AS. Point-of-care testing. In: Burtis CA, Ashwood ER, Bruns DE (Eds). Tietz textbook of clinical chemistry and molecular diagnostics. $4^{\text {th }}$ edition Philadelphia: Elsevier- Saunders Inc. 2006; 299-320.

7. Hudson MP, Christenson RH, Newby LK, et al. Cardiac markers: point of care testing. Clin Chim Acta 1999; 284: 223-37.

8. Price CP. Point-of-Care Testing for Cardiac Markers: Applications and Outcomes. Point of Care 2008;7(4): 261-265.

9. Awtry EH, Locscalo J. Coronary heart disease. In: Capenter $\mathrm{C}$; Griggs RC; and Locscalo J. CECEL Essential of Medicine. 6th ed. Philadelphia: ElsevierSaunders Inc. 2004; P 87-173.

10. Larsson $H$, Bergund $G$, Lindgard $\mathrm{F}$, et al. Comparison of ADA and WHO criteria for diagnosis of diabetes and glucose intolerance. Diabetologia 1998; 41:1124-5.

11. Wood D, Durrington P, Poulter N. Joint British recommendations on prevention of coronary heart disease in clinical practice. Heart 1998; 80 (Suppl.2): 1-29.

12. Selwyn AP, Braunwald E. Ischemic Heart Disease. In: Braunwald E; Fauci AS (Eds). Harrison's Principles of Internal Medicine. $15^{\text {th }}$ edition. New York: McGraw-Hill. 200; P 1399-410.

13. Innasimutthu AL, Siddhi SS, Rao GKB. Clinical presentation and ECG changes - how good is it in diagnosing troponin positive acute coronary syndrome. Anatol J Cardiol 2007;7 Supp 1:168-70.

14. hiu WK, Chan SH, Cheng CK, et al. Troponin I, myoglobin, and mass concentration of creatine kinase-MB in acute myocardial infarction. Q J M 1999; 92:711718.

15. Straface AL, Myers JH, Kirchick HJ, et al. A Rapid Point-of-Care Cardiac Marker Testing Strategy Facilitates the Rapid Diagnosis and Management of Chest Pain Patients in the Emergency Department. Ann Clin Pathol 2008; 129(5):788-95.

16. Panteghini $M$, Renze B. Enzymes. In: Tietz Fundamentals of Clinical Chemistry. Burtin CA and Ashwood ER (Eds). $6^{\text {th }}$ edition. Philadelphia: Saunders. 2008; P 320.

17. Uotila M, Ruoslathi E, Engvall E. Two-site sandwich enzyme immunoassay with monoclonal antibodies to human alpha fetoprotein. J Immunol Methods 1981;42: 11-5.

18. Cummins B, Auckland ML, Cummins. Cardiac-specific troponin-I radioimmunoassay in the diagnosis of acute myocardial infarction. Am. Heart J 1987;113:1333-44.

19. Viera AJ, Garrett JM. Understanding inters observer agreement: the Kappa statistic. Fam Med 2005; 37:360-3.

20. Hammett-Stabler CA, Nichols JS. Point-of-Care Testing, a Critical Component of Laboratory Medicine. Clin Biochem 2009; 42:135-41.

21. McCord J, Nowak RM, McCullough PA, et al. Ninetyminute exclusion of acute myocardial infarction by use of quantitative point-of-care testing of myoglobin and troponin I. Circulation 2001;104(13):1483-8.

22. Newby K, Storrow A, Gibler B, et al. Bed side multimarker testing for risk stratification in chest pain units. The chest pain evaluation by creatine kinase-MB, myoglobin, and troponin I (CHECKMATE) 
study. Circulation 2001; 103(14):1832-37.

23. Hamm CW, Goldmann BU, Heeschen C. Emergency room triage of patients with acute chest pain by means of rapid testing for cardiac troponin $\mathrm{T}$ or troponin $\mathrm{I}$. $\mathrm{N}$ Engl Med J 1997; 337 (23):1648-53.

24. Penttila K, Koukkunen H, Kemppainen A, et al. Myoglobin, creatine kinase $\mathrm{MB}$, troponin $\mathrm{T}$, and troponin I - rapid bedside assays in patients with acute chest pain. Int $\mathrm{J}$ Clin Lab Res 1999;29(2):93-101.

25. Baheti R, Laddha P, Gehlot RS. Value of Troponin $\mathrm{T}$ in the Diagnosis of Acute Myocardial Infarction. J I A $\mathrm{C} \quad \mathrm{M}$ 2002;3(1):55-8.

26. Kratz A, Lewandrowski KB, McDermott $S$, et al. Performance of a point-of-care qualitative triple cardiac marker screen under controlled laboratory conditions and in an emergency department setting. Clin Chim Acta 2002;325:79-85.

27. Guo X, Feng J, Guo H. The predictive value of the bedside troponin $\mathrm{T}$ test for patients with acute chest pain. Exp Clin Cardiol 2006;11(4):298-301.

28. Sanchez BG, Jarero SF, Teran $\mathrm{BB}$, et al. Qualitative determination of markers of myocardial necrosis during prehospital admission for acute coronary syndromes. Cir Cir 2006;74(4):231-5.

29. Luiz T, Ellinger $K$, Budde A. Evaluation of a rapid qualitative test for cardiac troponin $\mathrm{T}$ in clinical diagnosis of patients with acute coronary syndrome. Z Kardiol 1998;87(4):267-75. 